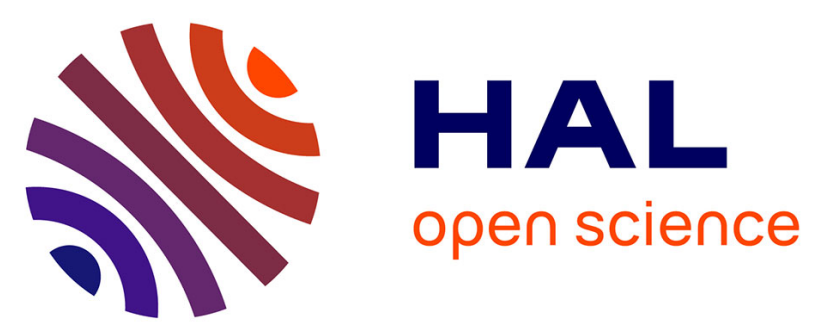

\title{
Independent dose verification system with Monte Carlo simulations using TOPAS for passive scattering proton therapy at the National Cancer Center in Korea
}

Wook-Geun Shin, Mauro Testa, Hak Soo Kim, Jong Hwi Jeong, Se Byeong Lee, Yeon-Joo Kim, Chul Hee Min

\section{To cite this version:}

Wook-Geun Shin, Mauro Testa, Hak Soo Kim, Jong Hwi Jeong, Se Byeong Lee, et al.. Independent dose verification system with Monte Carlo simulations using TOPAS for passive scattering proton therapy at the National Cancer Center in Korea. Phys.Med.Biol., 2017, 62 (19), pp.7598-7616. 10.1088/1361-6560/aa8663 . hal-01763848

\section{HAL Id: hal-01763848 \\ https://hal.science/hal-01763848}

Submitted on 8 Nov 2018

HAL is a multi-disciplinary open access archive for the deposit and dissemination of scientific research documents, whether they are published or not. The documents may come from teaching and research institutions in France or abroad, or from public or private research centers.
L'archive ouverte pluridisciplinaire HAL, est destinée au dépôt et à la diffusion de documents scientifiques de niveau recherche, publiés ou non, émanant des établissements d'enseignement et de recherche français ou étrangers, des laboratoires publics ou privés. 
PAPER

\section{Independent dose verification system with Monte Carlo simulations using TOPAS for passive scattering proton therapy at the National Cancer Center in Korea}

To cite this article: Wook-Geun Shin et al 2017 Phys. Med. Biol. 627598

\section{Manuscript version: Accepted Manuscript}

Accepted Manuscript is "the version of the article accepted for publication including all changes made as a result of the peer review process, and which may also include the addition to the article by IOP Publishing of a header, an article ID, a cover sheet and/or an 'Accepted

Manuscript' watermark, but excluding any other editing, typesetting or other changes made by IOP Publishing and/or its licensors"

This Accepted Manuscript is (c) (c) 2017 Institute of Physics and Engineering in Medicine.

During the embargo period (the 12 month period from the publication of the Version of Record of this article), the Accepted Manuscript is fully protected by copyright and cannot be reused or reposted elsewhere.

As the Version of Record of this article is going to be / has been published on a subscription basis, this Accepted Manuscript is available for reuse under a CC BY-NC-ND 3.0 licence after the 12 month embargo period.

After the embargo period, everyone is permitted to use copy and redistribute this article for non-commercial purposes only, provided that they adhere to all the terms of the licence https://creativecommons.org/licences/by-nc-nd/3.0

Although reasonable endeavours have been taken to obtain all necessary permissions from third parties to include their copyrighted content within this article, their full citation and copyright line may not be present in this Accepted Manuscript version. Before using any content from this article, please refer to the Version of Record on IOPscience once published for full citation and copyright details, as permissions will likely be required. All third party content is fully copyright protected, unless specifically stated otherwise in the figure caption in the Version of Record.

View the article online for updates and enhancements. 


\title{
Independent dose verification system with Monte Carlo simulations using TOPAS for passive scattering proton therapy at the National Cancer Center in Korea
}

\author{
Wook-Geun Shin ${ }^{1}$, Mauro Testa ${ }^{2}$, Hak Soo Kim ${ }^{3}$, \\ Jong Hwi Jeong ${ }^{3}$, Se Byeong Lee ${ }^{3}$, Yeon-Joo Kim ${ }^{3}$, \\ Chul Hee Min ${ }^{1 *}$ \\ ${ }^{1}$ Department of Radiation Convergence Engineering, Yonsei University, Wonju, Korea \\ ${ }^{2}$ Institute of Nuclear Physics of Lyon, Villeurbanne, France \\ ${ }^{3}$ Proton Therapy Center, National Cancer Center, Goyang, Korea \\ E-mail: chmin@yonsei.ac.kr
}

February 2014

\begin{abstract}
For the independent validation of treatment plans, we developed a fully automated Monte Carlo (MC)-based patient dose calculation system with the tool for particle simulation (TOPAS) and proton therapy machine installed at the National Cancer Center in Korea (KNCC) to enable routine and automatic dose recalculation for each patient. The proton beam nozzle was modeled with TOPAS to simulate the therapeutic beam, and MC commissioning was performed by comparing percent depth dose with the measurement. The beam set-up based on the prescribed beam range and modulation width was automated by modifying the vendor-specific method. The CT phantom was modeled based on the DICOM CT files with TOPAS-built-in function, and an in-house-developed $\mathrm{C}++$ code directly imports the $\mathrm{CT}$ files for positioning the CT phantom, RT-plan file for simulating the treatment plan, and RT-structure file for applying the Hounsfield unit (HU) assignment, respectively. The developed system was validated by comparing the dose distributions with those calculated by the treatment planning system (TPS) for a lung phantom and two patient cases of abdomen and internal mammary node. The results of the beam commissioning were in good agreement of up to $0.8 \mathrm{~mm}^{2} / \mathrm{g}$ for $\mathrm{B} 8$ option in both of the beam range and the modulation width of the spread-out Bragg peaks. The beam set-up technique can predict the range and modulation width with an accuracy of $0.06 \%$ and $0.51 \%$, respectively, with respect to the prescribed range and modulation in arbitrary points of B5 option (128.3, 132.0, and $141.2 \mathrm{~mm}^{2} / \mathrm{g}$ of range). The dose distributions showed higher than $99 \%$ passing rate for the 3D gamma index (3 $\mathrm{mm}$ distance to agreement and $3 \%$ dose difference) between the MC simulations and the clinical TPS in the target volume. However, in the normal tissues, less favorable agreements were obtained for the radiation treatment planning with the lung phantom and internal mammary node cases. The discrepancies might come from the limitations of the clinical TPS, which is the inaccurate dose calculation algorithm for the scattering effect, in the range compensator and inhomogeneous material. Moreover, the steep slope of the compensator, conversion of the $\mathrm{HU}$ values to the human phantom, and the dose
\end{abstract}


calculation algorithm for the HU assignment also could be reasons of the discrepancies. The current study could be used for the independent dose validation of treatment plans including high inhomogeneities, the steep compensator, and riskiness such as lung, head $\&$ neck cases. According to the treatment policy, the dose discrepancies predicted with MC could be used for the acceptance decision of the original treatment plan.

\section{Introduction}

The number of proton therapy facilities worldwide is currently doubling every five years and approximately above 130,000 cancer patients had been treated with proton therapy until end of 2015 (Martin 2016). The physical characteristic of the proton beam, called the Bragg peak, enable proton therapy to deliver a high conformal target dose without delivering an exit dose beyond the target. To fully utilize the characteristics of the proton beam, the patient dose distribution must be predicted as precisely as possible with radiation treatment planning (RTP). Pencil-beam algorithms (PBAs) commercially employed in RTP for proton therapy are analytical models based on dose kernels measured in a water phantom. However, PBAs are known to exhibit limited accuracy for dose calculations in inhomogeneous materials mainly because of the scattering effect (Paganetti 2012). To overcome the limitations of conventional PBAs, several studies have proposed the investigation of the collapse cone convolution (CCC) algorithm (Ahnesjö 1989), the anisotropic analytical algorithm (AAA) (Van Esch et al. 2006), the simplified Monte Carlo (SMC) (Jia et al. 2010), neural networks (Lee et al. 2015), and other techniques that use an analytical model. Nevertheless, Monte Carlo (MC) methods are considered to be the most reliable tool for patient dose calculation in radiation therapy because they simulate all the interactions between particles and matter for each event (Paganetti et al. 2008)(Cheong et al. 2004). The tool for particle simulation (TOPAS), which is based on Geometry and tracking4 (Geant4) and specifically dedicated to radiotherapy applications (Perl et al. 2012)(Agostinelli et al. 2003), and MC-AUTO have been suggested to automatically integrate the MC calculation processes (Verburg et al. 2016). To simulate therapeutic proton beam of precise range and modulation width, a vendor-specific beam set-up algorithm (Conversion algorithm, Convalgo-IBA) converting the range and modulation width to the nozzle set-up is provided. However, the beam range and modulation width provided by Convalgo do not always agree with the physical range and width that should be delivered to the patient to comply with the prescribed dose in the target volume (Rah et al. 2014). Therefore, patient-specific quality assurance (QA) is performed to calibrate the beam set-up based on physical measurements, which is a time-consuming process lasting approximately 2 hours (Kooy et al. 2005). The aim of this study is to develop a fully automated system including the accurate prediction of the range and modulation width for the independent patient dose validation for passive scattering proton therapy with MC simulation using TOPAS. At the National Cancer Center in Korea (KNCC), we modeled an IBA universal nozzle (Ion Beam Application SA, Louvain-La-Neuve, 
Belgium) to simulate therapeutic beams and developed a technique to calibrate a vendorspecific conversion algorithm for determination of beam set-up. The dose validation for the developed system was performed by comparing the dose distribution measured with an ionization chamber. The patient doses calculated by MC and Eclipse RTP (Varian Medical System, Palo Alto, CA) were compared and analyzed with a lung phantom, an abdomen (ABD), and an internal mammary node (IMN) cases.

\section{Materials and methods}

\subsection{MC modeling of the proton beam nozzle}

To recalculate patient dose with the MC method, accurate modeling and commissioning of the therapeutic beam nozzle must be performed. The passive scattering mode of the proton beam nozzle (Proteus235, IBA) installed at the KNCC was modeled and validated based on the detailed manufacturers blueprint and a Geant4 code developed in a previous study (Shin et al. 2015). TOPAS (2.0.p03 version) was employed with various dose calculation functions provided for MC simulations of proton therapy.

Figure 1(a) shows the proton beam nozzle, as modeled in TOPAS. The beam nozzle simulations were performed without the proton accelerator; therefore, the energy spread, spot size, and angular spread of the initial proton beam exiting the cyclotron were benchmarked by measurements performed in a previous study at KNCC (Shin et al. 2010). The IBA universal nozzle consisted of the first beam monitoring device (IC1), the first scatterer (FS), the range modulation wheel (RMW), the second scatterer (SS), the second beam monitoring device (IC2), the variable collimator (VC), the snout (SNT), and patient-specific components (PSCs), including an aperture and a compensator. We benchmarked the same work of IBA machine modeling (Testa et al. 2013). The RMW in the IBA model is the main component generating the spreadout Bragg peak (SOBP) by sequential changing of the beam range. The RMW was modeled based on the blueprint with three small wheels enclosed in a large wheel, and each small wheel includes three tracks. The pristine proton beam is passing through the step of the different thickness in the RMW during the wheel rotation. In the figure 1(b), start digit is the rotational position of the beam spot when a beam commences and the stop digit is the position of beam end. The stop digit was typically controlled for the modifying the modulation width of SOBP in passive scattering proton therapy. However, the red and the blue tracks, which indicates the different start digit, include first step to $7^{\text {th }}$ and $10^{\text {th }}$ step, respectively. Because of the machine set-up, the difference of the start digit could be change not only modulation width but also range and uniformity of the SOBP, so the zero angle calibration is crucial. In this study, the zero angles were calibrated according to the SOBP tendency analysis (Beltran et al. 2014). The PSCs attached downstream of the SNT were modeled by importing the patient-specific aperture and compensator from the digital imaging and communications in medicine (DICOM) file (NEMA 2009). For the beam nozzle simulations, the physics model 


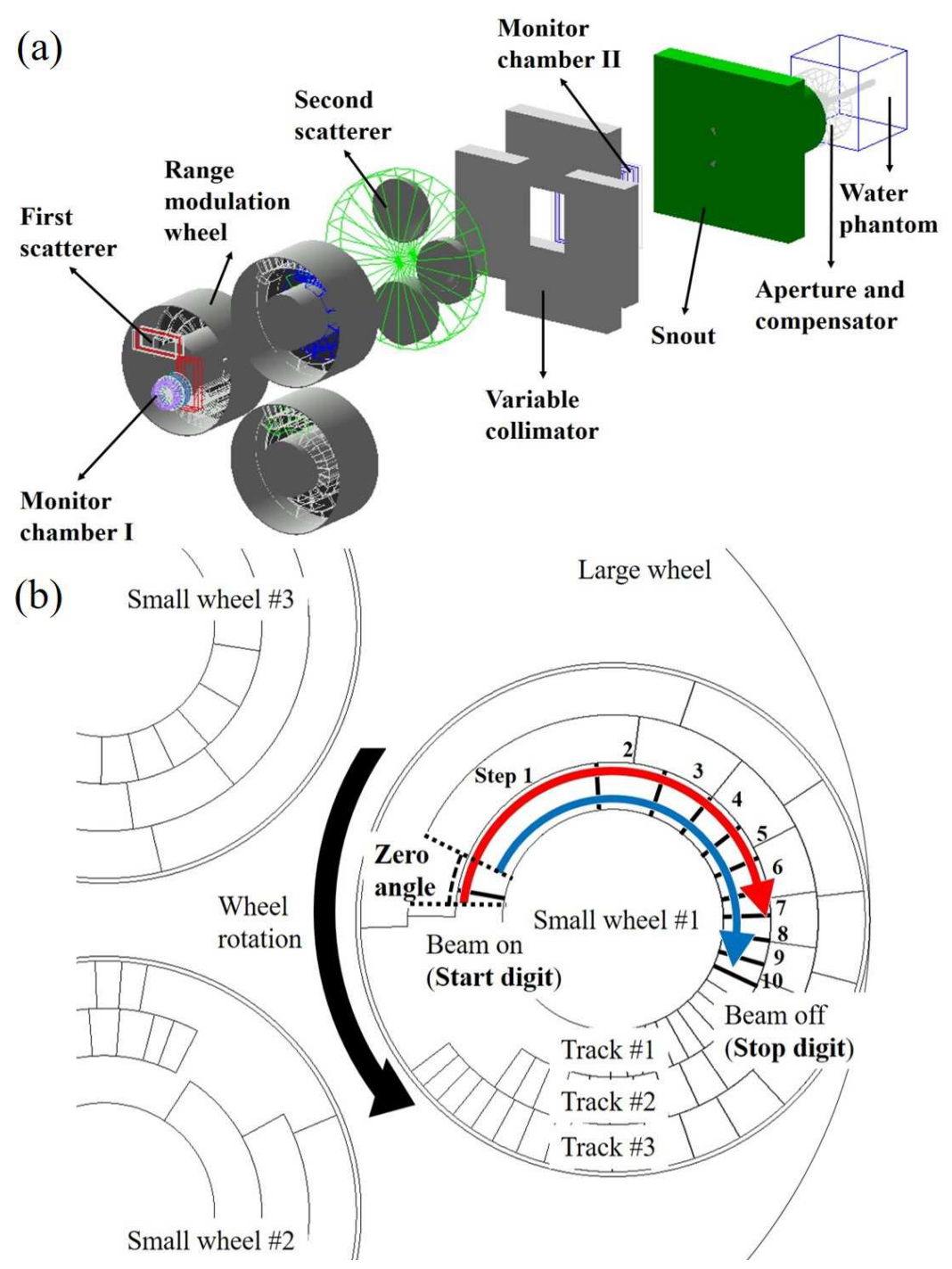

Figure 1. (a) A schematic illustration of the proton beam nozzle and a water phantom modeled by TOPAS. The initial proton beam is directed from left to right. The central line in the water phantom (gray line) indicates the scoring volume used to calculate the percent depth dose. (b) A schematic illustration of the range modulation wheel (RMW), consisting of three small wheels and nine tracks, as modeled within TOPAS. The beam would be entering into the page. The circles and arrows indicate the positions that the beam irradiation is started and the irradiated tracks before the zero angle calibration (red) and with zero angle calibration (blue).

employed G4EM-Standard_opt4 for electromagnetic processes, G4Decay for radioactive decay processes, G4h-Elastic for elastic hadron processes, G4h-QGSP_BIC_HP for inelastic processes, G4Ion-Binarycascade for ion physics, and G4Stopping for stopping powers (Jarlskog \& Paganetti 2008). For the MC simulations, the beam commissioning was performed by comparing the percent depth doses (PDDs) calculated with TOPAS and the PDDs measured with a Markus ionization chamber (PTW, Freiburg, Germany). The dimensions of the box-shaped water phantom were $30 \times 30 \times 30 \mathrm{~cm}^{3}$; to calculate the PDDs, 300 cylindrical slabs with $1 \mathrm{~cm}$ diameter and $0.1 \mathrm{~cm}$ thickness were modeled 
Table 1. Ranges and modulation widths measured with a Markus ionization chamber and the beam set-up for the eight beam options employed for the MC commissioning.

\begin{tabular}{lllllllll}
\hline Beam & $\begin{array}{l}\text { Convalgo } \\
\text { option } \\
\left(\mathrm{mm}^{2} \mathrm{~g}^{-1}\right)\end{array}$ & $\begin{array}{l}\text { Convalgo } \\
\text { modulation } \\
\text { width } \\
\left(\mathrm{mm}^{2} \mathrm{~g}^{-1}\right)\end{array}$ & $\begin{array}{l}\text { Measured } \\
\text { range } \\
\left(\mathrm{mm}^{2} \mathrm{~g}^{-1}\right)\end{array}$ & $\begin{array}{l}\text { Measured } \\
\text { modulation } \\
\text { width } \\
\left(\mathrm{mm}^{2} \mathrm{~g}^{-1}\right)\end{array}$ & $\begin{array}{l}\text { Range } \\
\text { modulation } \\
\text { track }\end{array}$ & $\begin{array}{l}\text { Beam } \\
\text { energy } \\
(\mathrm{MeV})\end{array}$ & $\begin{array}{l}\text { Start } \\
\text { angle } \\
(\text { degree })\end{array}$ & $\begin{array}{l}\text { Stop } \\
\text { angle } \\
(\text { degree })\end{array}$ \\
\hline B1 & 47.3 & 21.5 & 49.1 & 21.5 & $\# 7$ & 159.18 & -4.2 & 128 \\
B2 & 61.1 & 27.8 & 62.0 & 29.5 & $\# 7$ & 166.91 & -4.2 & 142 \\
B3 & 76.8 & 34.9 & 78.9 & 35.4 & $\# 4$ & 157.73 & 0 & 179 \\
B4 & 97.4 & 44.3 & 100.1 & 48.7 & $\# 5$ & 170.89 & +4.2 & 224 \\
B5 & 125.6 & 57.1 & 129.3 & 59.5 & $\# 4$ & 166.82 & 0 & 212 \\
B6 & 167.5 & 76.1 & 171.5 & 79.5 & $\# 5$ & 189.87 & +4.2 & 263 \\
B7 & 210.7 & 95.8 & 213.4 & 92.3 & $\# 6$ & 213.33 & -4.2 & 295 \\
B8 & 240.1 & 102.1 & 242.5 & 103.4 & $\# 8$ & 210.63 & -5.6 & 281 \\
\hline
\end{tabular}

along the center line of the water phantom. Both of the measurements and MC simulations employed a snout with $250 \mathrm{~mm}$ diameter (SNT250), and the water phantom was positioned at a face of the snout. The beam nozzle of the KNCC covers the proton beam range of $4.51-28.42 \mathrm{~g} / \mathrm{cm}^{2}$, and the manufacturer categorized the proton beams to 8 options according to the beam range (Lin et al. 2014). The 8 proton beams arbitrary selected for each beam option were employed for beam commissioning, and table 1 shows the ranges, the modulation widths, and the beam set-up of eight beam options.

\subsection{Determination algorithm for beam set-up}

The conversion algorithm (Convalgo, IBA) determines the beam set-up, such as a disk of the scatterer, a track of the RMW, and the beam energy, according to the range and the modulation width of a SOBP. The beam energy and the stop digit mainly determining the beam range and the modulation width were predicted based on an exponential function and $4^{\text {th }}$ order functions, respectively shown in (1) and (2).

$$
\begin{aligned}
& E(R)=e^{c_{0}+c_{1} \ln R+c_{2}(\ln R)^{2}+c_{3}(\ln R)^{3}} \\
& S D_{B O}(m)=C_{B O, 0}+C_{B O, 1} m+C_{B O, 2} m^{2}+C_{B O, 3} m^{3}+C_{B O, 4} m^{4}
\end{aligned}
$$

Where $E(R)$ and $S D_{B O}(m)$ indicate the initial beam energy to obtain a beam range of $R$, and stop digit to obtain a modulation width of $m$, respectively. The both of $c$ and $C$ are constants, and the constants for predicting modulation width $C$ depend on beam option $B O$. However, the functions fitting the relationship between initial energy and beam range, and the relationship between stop digit and modulation width do not fully consider the physical phenomenon such as the energy dependency of the modulation width. Because of the above reason, it was reported that the Convalgo potentially increase the errors in the range and modulation width up to $4 \mathrm{~mm}^{2} / \mathrm{g}$ in comparison to 


\section{Independent dose verification system with Monte Carlo simulations using TOPAS}

the measurements (Kim et al. 2011). Due to these errors, a time-consuming empirical calibration of the beam set-up is required during the pretreatment QA in clinics. For the accurate MC simulation, the identical range and modulation width with the delivered beam to the patient should be simulated. In the case of the range calibration, the maximum and minimum ranges of the eight beam options were assessed and linearly interpolated based on the assumption that the Convalgo and $\mathrm{MC}$ ranges are linearly correlated within each beam option. The modulation width in Convalgo was derived by a $4^{\text {th }}$-order function describing the relationship between the modulation width and the stop digit in the RMW. However, even when the beam option and the stop digit were the same, the modulation widths could be different according to ranges because of the energy dependence of the modulation width. In this study, stop-digit-to-modulationwidth (SD-MW) curves of the maximum and minimum ranges were calculated for each beam option using MC simulations; these curves were linearly interpolated based on the residual energy of the proton beams as follows:

$$
S D(R, m)=S D\left(R_{\text {min }}, m\right)+\frac{\Delta S D_{B O}}{\Delta E}\left[E(R)-E\left(R_{\text {min }}\right)\right]
$$

where $S D(R, m)$ is the stop digit in the RMW produced in the range $R$ and the modulation width $m$, and $E(R)$ is the residual energy of the proton range $R$, and $\triangle S D_{B O}$ and $\Delta E$ is the stop digit and energy difference between maximum and the minimum ranges $R_{\max }$ and $R_{\text {min }}$ in the beam option $B O$. Equation (3) expresses that $S D(R, m)$ is a linear function of the residual energy $E(R)$. In (3), the gradient of the linear function in the specific beam option $B O$ is:

$$
\frac{\Delta S D_{B O}}{\Delta E}=\frac{S D\left(R_{\max }, m\right)-S D\left(R_{\min }, m\right)}{E\left(R_{\max }\right)-E\left(R_{\min }\right)}
$$

Therefore, the linear function between the SD-MW curves and the modulation widths is provided by

$$
S D(R, m)=S D\left(R_{\min }, m\right)+\frac{S D\left(R_{\max }, m\right)-S D\left(R_{\min }, m\right)}{\left(E\left(R_{\max }\right)-E\left(R_{\min }\right)\right.}\left[E(R)-E\left(R_{\min }\right)\right]
$$

Finally, (5) was employed to assess the SD-MW curve for a prescribed range $R$. The relationship between the prescribed range $R$ and the residual energy $E(R)$ was benchmarked by NIST measurements (Berger 1999). The corresponding formula is

$$
E(R)=\frac{R}{0.002527}^{\frac{1}{1.742}}
$$

To automatically analyze the results of the MC simulations, an in-house developed algorithm based on Matrix Laboratory (MATLAB) was employed (MathWorks 2005). To verify the feasibility of the assumptions, the ranges and modulation widths derived with the algorithm were compared with those calculated by $\mathrm{MC}$ and Convalgo methods. The developed algorithm determines the beam set-up for certain input parameters of the range and modulation width, and it was implemented as a dose calculation system for an automatic procedure. 


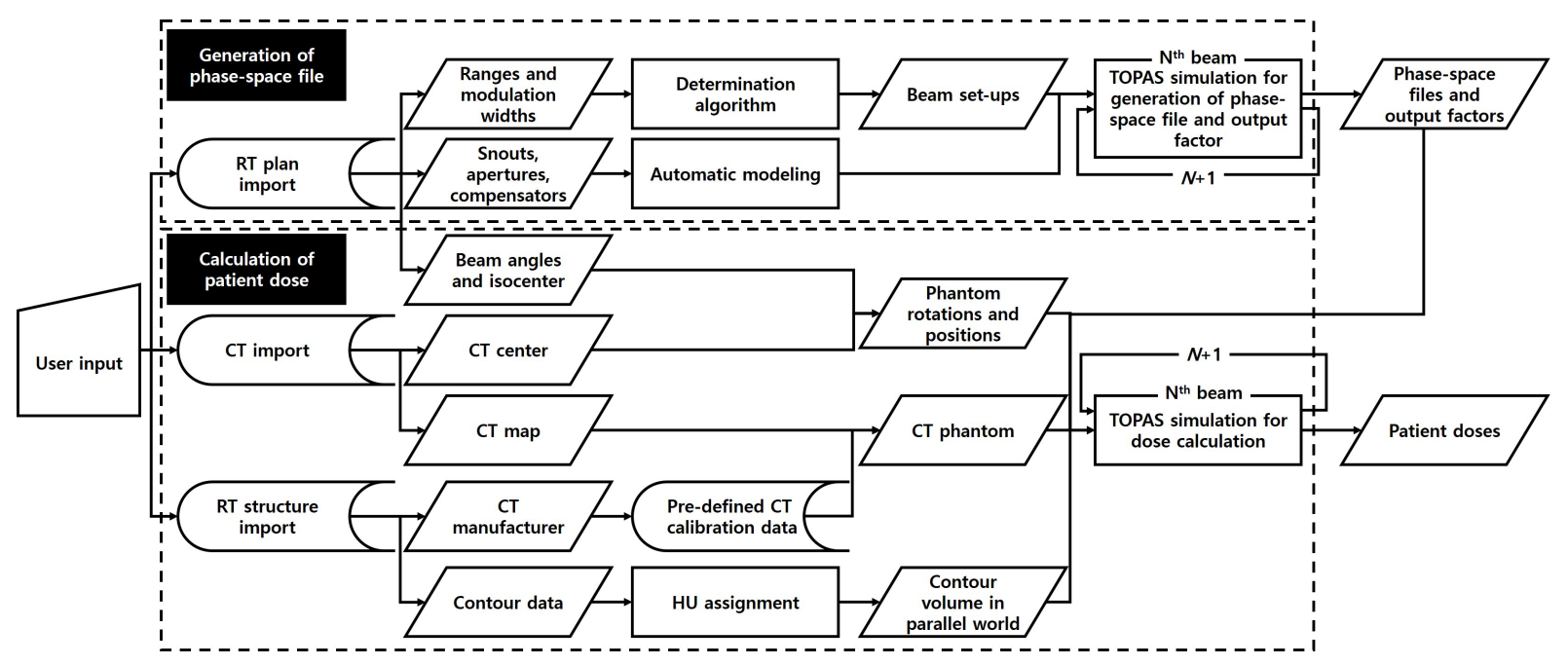

Figure 2. Flowchart for patient dose calculation using TOPAS. The upper section is for recording phase-space file and the lower section is for patient dose calculation.

\subsection{Streamlined $M C$ workflow for patient dose calculation.}

Figure 2 illustrates the procedure of the dose calculation system. First, the determination algorithm controls the beam set-up according to the prescribed range and modulation width. Using the beam set-up, a phase-space file is recorded at the end of the beam nozzle, and the output factor is simultaneously calculated in the virtual water phantom. The phase-space file includes the energy, position, and direction of neutrons and protons passing through the virtual plate downstream of the compensator. The other particles, such as electrons and heavy particles, are not considered in the current study because of their negligible contribution to the target volume, but taking long calculation time. The recorded phase-space file of therapeutic beam passing through the proton beam nozzle could be employed several times. The reuse of the phase-space file could improve the statistics without the simulation time in the beam nozzle.

One of the most important parts of the second step is the automatic modeling of the aperture and compensator by importing the patient DICOM file. The aperture and compensator are automatically modeled based on arbitrary polygons containing brass and poly-methyl methacrylate (PMMA), respectively. However, Eclipse RTP exports the aperture and compensator data normalized to the isocenter. That is, the position data must be calibrated to consider the divergence of the proton beam, as shown in figure 3. Milling tool in KNCC applies the calibration based on the treatment planning, and we replicate the calibration to the automatic modeling of the PSCs. To assess the beam divergence, the position of each PSC point $(x, y)$ was calibrated by using the ratio of the virtual-source-to-isocenter distance $(S I D)$, isocenter-to-aperture-tray distance $(I A D)$, and isocenter-to-compensator-tray distance $(I C D)$.

$$
(x, y)_{c a l}=(x, y) \times\left(1-\frac{I A D, I C D}{S I D}\right)
$$

To verify the validity of the automatically modeled PSCs, the dose distribution 


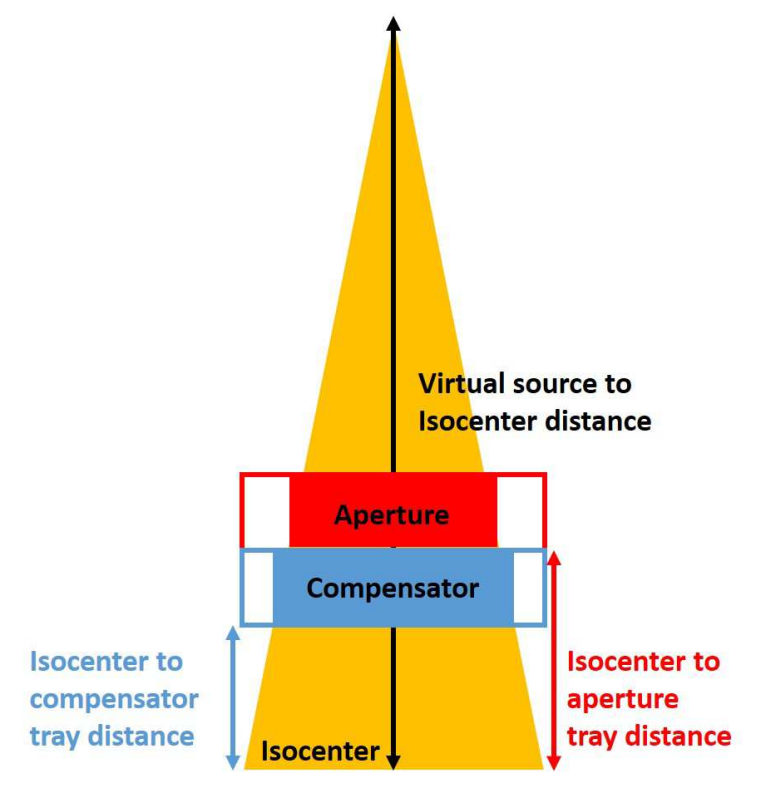

Figure 3. The relationship between the virtual-source-to-isocenter distance (black arrow), isocenter-to-aperture-tray distance (red arrow), isocenter-to-compensator-tray distance (blue arrow), PSCs normalized to the isocenter (larger, red and blue boxes), and calibrated PSCs (smaller, solid red and blue boxes).

calculated by Eclipse RTP in a virtual water phantom was compared with that of the TOPAS simulation. For the patient dose calculation, a computed tomography (CT) image was converted to the computational voxel phantom by using the Schneider method in the RTP (Schneider et al. 1996). In this study, we followed the procedure to convert the Hounsfield unit (HU), which represents electron density, to the chemical composition and mass density (Schneider et al. 2000). First, a calibration was performed for the CT installed at the KNCC with measurements of a CIRS phantom (Model 062M, CIRS Inc., Norfolk, VA) (Cheng 2008). Based on the elementary compositions of the human tissue in reference paper (White et al. 1987), tables were generated for mass density and the material compositions as a function of the HU as shown in figure 4 and table 2, respectively. Finally, to simplify the simulations, the CT phantom was rotated and moved depending on the beam angle and isocenter from the patient DICOM file instead of moving all the components in the beam nozzle.

For specific cases in which the material and the following $\mathrm{HU}$ value is different from the general tissues employing in the patient treatment such as the contrast agent, fiducial marker, and couch, the correction of the discrepancy between CT simulation and treatment should be applied. HU assignment which replaces the HU values of the undesired voxels to the corrected HU values is typically used in clinical RTP. To apply HU assignment in the MC simulations, the HU values in the contour volume were replaced to the assigned HU values; however, this method is not suitable for routine procedures. In this study, LayeredMassGeometry function originally developed for seed simulations in brachytherapy was employed. This function automatically models 


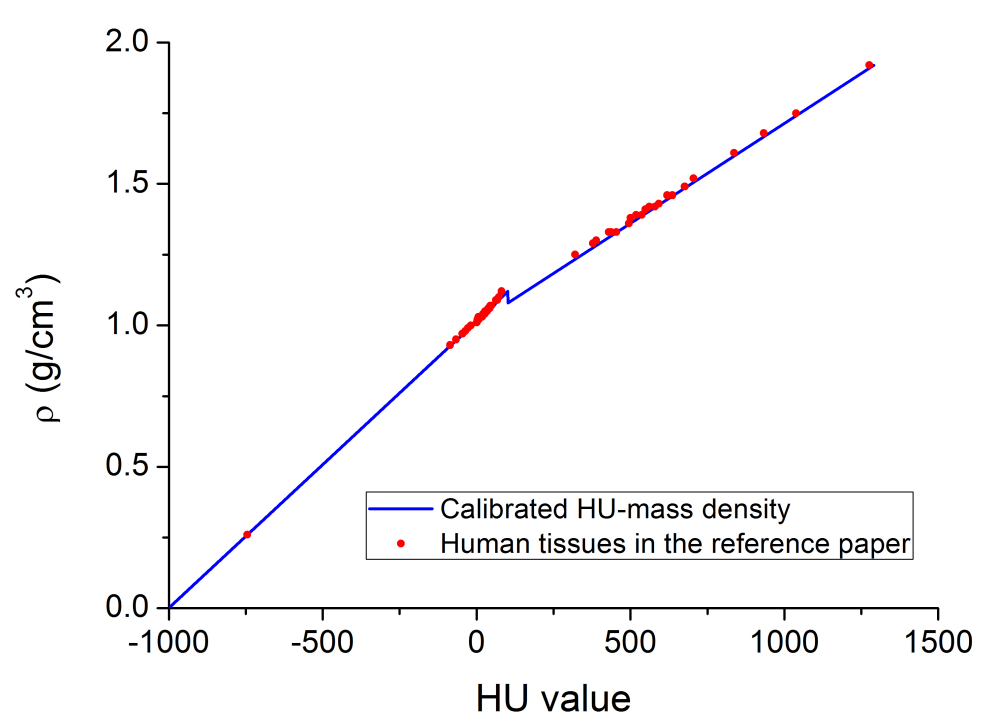

Figure 4. The calibrated curve of the HU to the mass density (blue solid line) and the human tissue in the reference paper (red dots).

the contour volume using the G4ExtrudedSolid function and projected it on the CT phantom as a parallel world (Enger et al. 2012).

\subsection{Case study}

To verify the feasibility of the dose calculation system, a lung phantom, an internal mammary node (IMN), and an abdomen (ABD) cases were employed. Table 3 shows the ranges and modulation widths of the RTP and MC for each beam field. All of the PDDs were well matched with an RTP within $1 \mathrm{~mm}^{2} / \mathrm{g}$ in range and modulation width in the water phantom.

Unlike commercial RTPs, the MC directly calculated the dose-to-medium using the HU-material conversion table. In the high $\mathrm{HU}$ value region, the dose-to-medium and the dose-to-water have been reported to be similar (Paganetti 2009). However, the dose distributions calculated in this study were converted to the dose-to-water using a function provided by TOPAS to quantitatively compare them with the dose distribution calculated by RTP (Almond et al. 1999). To quantitatively compare the two dose distributions, the amount of the dose delivered to the target volume must be suitably matched between the treatment and the MC calculation. The dose scaling factor (DSF) is typically employed for both the MC and RTP calculations because the large number of particles used in clinics involves difficulties related to the burden of the calculation time. The DSF is a multiplication coefficient that adapts the calculated dose to the prescribed dose. A pre-calculated output factors was used to determine the DSF as

$$
D S F=\frac{\text { prescription dose }(G y)}{O F(G y) \times n \text { Particles }}
$$


Independent dose verification system with Monte Carlo simulations using TOPAS 10

Table 2. The calibration table for converting the $\mathrm{HU}$ value to the chemical composition of human tissue

\begin{tabular}{|c|c|c|c|c|c|c|c|c|c|c|c|c|c|}
\hline \multirow{2}{*}{\multicolumn{2}{|c|}{ HU value }} & \multicolumn{12}{|c|}{$w_{i}(\%)$} \\
\hline & & $\mathrm{H}$ & $\mathrm{C}$ & $\mathrm{N}$ & $\mathrm{O}$ & $\mathrm{Na}$ & $\mathrm{Mg}$ & $\mathrm{P}$ & S & $\mathrm{Cl}$ & $\mathrm{Ar}$ & $\mathrm{K}$ & $\mathrm{Ca}$ \\
\hline-1000 & -951 & 0.0 & 0.0 & 75.5 & 23.2 & 0.0 & 0.0 & 0.0 & 0.0 & 0.0 & 1.3 & 0.0 & 0.0 \\
\hline-952 & -97 & 10.3 & 10.5 & 3.1 & 74.9 & 0.2 & 0.0 & 0.2 & 0.3 & 0.3 & 0.0 & 0.2 & 0.0 \\
\hline-98 & -68 & 11.6 & 68.1 & 0.2 & 19.8 & 0.1 & 0.0 & 0.0 & 0.1 & 0.1 & 0.0 & 0.0 & 0.0 \\
\hline-69 & -41 & 11.3 & 56.7 & 0.9 & 30.8 & 0.1 & 0.0 & 0.0 & 0.1 & 0.1 & 0.0 & 0.0 & 0.0 \\
\hline-42 & -13 & 11.0 & 45.8 & 1.5 & 41.1 & 0.1 & 0.0 & 0.1 & 0.2 & 0.2 & 0.0 & 0.0 & 0.0 \\
\hline-14 & 12 & 10.8 & 35.6 & 2.2 & 50.9 & 0.0 & 0.0 & 0.1 & 0.2 & 0.2 & 0.0 & 0.0 & 0.0 \\
\hline 13 & 19 & 10.6 & 28.4 & 2.6 & 57.8 & 0.0 & 0.0 & 0.1 & 0.2 & 0.2 & 0.0 & 0.1 & 0.0 \\
\hline 20 & 88 & 10.3 & 13.4 & 3.0 & 72.3 & 0.2 & 0.0 & 0.2 & 0.2 & 0.2 & 0.0 & 0.2 & 0.0 \\
\hline 89 & 123 & 9.4 & 20.7 & 6.2 & 62.2 & 0.6 & 0.0 & 0.0 & 0.6 & 0.3 & 0.0 & 0.0 & 0.0 \\
\hline 124 & 166 & 9.5 & 45.5 & 2.5 & 35.5 & 0.1 & 0.0 & 2.1 & 0.1 & 0.1 & 0.0 & 0.1 & 4.5 \\
\hline 167 & 248 & 8.9 & 42.3 & 2.7 & 36.3 & 0.1 & 0.0 & 3.0 & 0.1 & 0.1 & 0.0 & 0.1 & 6.4 \\
\hline 249 & 331 & 8.2 & 39.1 & 2.9 & 37.2 & 0.1 & 0.0 & 3.9 & 0.1 & 0.1 & 0.0 & 0.1 & 8.3 \\
\hline 332 & 415 & 7.6 & 36.1 & 3.0 & 38 & 0.1 & 0.1 & 4.7 & 0.2 & 0.1 & 0.0 & 0.0 & 10.1 \\
\hline 416 & 499 & 7.1 & 33.5 & 3.2 & 38.7 & 0.1 & 0.1 & 5.4 & 0.2 & 0.0 & 0.0 & 0.0 & 11.7 \\
\hline 500 & 584 & 6.6 & 31.0 & 3.8 & 39.4 & 0.1 & 0.1 & 6.1 & 0.2 & 0.0 & 0.0 & 0.0 & 13.2 \\
\hline 585 & 668 & 6.1 & 28.7 & 3.5 & 40.0 & 0.1 & 0.1 & 6.7 & 0.2 & 0.0 & 0.0 & 0.0 & 14.6 \\
\hline 669 & 753 & 5.6 & 26.5 & 3.6 & 40.5 & 0.1 & 0.2 & 7.3 & 0.3 & 0.0 & 0.0 & 0.0 & 15.9 \\
\hline 754 & 836 & 5.2 & 24.6 & 3.7 & 41.1 & 0.1 & 0. & 7.8 & 0.3 & 0.0 & 0.0 & 0.0 & 17.0 \\
\hline 837 & 921 & 4.9 & 22.7 & 3.8 & 41.6 & 0.1 & 0. & 8.3 & 0.3 & 0.0 & 0.0 & 0.0 & 18.1 \\
\hline 922 & 1006 & 4.5 & 21.0 & 3.9 & 42.0 & 0.1 & 0.2 & 8.8 & 0.3 & 0.0 & 0.0 & 0.0 & 19.2 \\
\hline 1007 & 1090 & 4.2 & 19.4 & 4.0 & 42.5 & 0.1 & 0.2 & 9.2 & 0.3 & 0.0 & 0.0 & 0.0 & 20.1 \\
\hline 1091 & 1175 & 3.9 & 17.9 & 4.1 & 42.9 & 0.1 & 0.2 & 9.6 & 0.3 & 0.0 & 0.0 & 0.0 & 21.0 \\
\hline 1176 & 1260 & 3.6 & 16.5 & 4.2 & 43.2 & 0.1 & 0.2 & 10 & 0.3 & 0.0 & 0.0 & 0.0 & 21.9 \\
\hline 1261 & 1342 & 3.4 & 15.5 & 4.2 & 43.5 & 0.1 & 0.2 & 10.3 & 0.3 & 0.0 & 0.0 & 0.0 & 22.5 \\
\hline
\end{tabular}

where $O F$ is the output factor, defined as the average dose of a \pm 1 -cm region in the middle of the SOBP divided by the number of particles in $\mathrm{MC}$ simulation, and $n$ Particles is the number of particles used to calculate the patient dose, including the reuse of the phase-space file. Finally, the patient dose can be assessed by the multiplication of the MC dose with the DSF. The number of particles recorded in the phase-space file was $3 \times 10^{7}$ with $2 \times 10^{8}$ primary protons, and the phase-space file was reused 5 times that the criteria of the saturated statistics for the patient dose calculation to obtain sufficient statistical accuracy with the total of $10^{9}$ primary protons (Méndez et al. 2015). Calculation times of about 70 CPU-hours and 40 CPU-hours with Xeon E-2697v3 (Intel, Santa Clara, CA) of 2.6 GHz were required to record the phasespace file and to calculate the patient dose for each case, respectively. We employed 
Table 3. Ranges and modulation widths calculated by Eclipse (RTP) and TOPAS (MC) for each beam field of the three $\operatorname{cases}^{a}$

\begin{tabular}{lllllll}
\hline & $\begin{array}{l}\text { Range RTP } \\
\left(\mathrm{mm}^{2} \mathrm{~g}^{-1}\right)\end{array}$ & $\begin{array}{l}\text { Range MC } \\
\left(\mathrm{mm}^{2} \mathrm{~g}^{-1}\right)\end{array}$ & $\begin{array}{l}\text { Difference } \\
(\mathrm{MC}-\mathrm{RTP}) \\
\left(\mathrm{mm}^{2} \mathrm{~g}^{-1}\right)\end{array}$ & $\begin{array}{l}\text { Mod. RTP } \\
\left(\mathrm{mm}^{2} \mathrm{~g}^{-1}\right)\end{array}$ & $\begin{array}{l}\text { Mod. MC } \\
\left(\mathrm{mm}^{2} \mathrm{~g}^{-1}\right)\end{array}$ & $\begin{array}{l}\text { Difference } \\
(\mathrm{MC}-\mathrm{RTP}) \\
\left(\mathrm{mm}^{2} \mathrm{~g}^{-1}\right)\end{array}$ \\
\hline Phantom 1A & 121.6 & 122.1 & 0.5 & 71.3 & 69.1 & -0.2 \\
IMN 1A & 91.6 & 91.2 & -0.4 & 43.0 & 42.5 & -0.5 \\
IMN 1B & 80.2 & 80.0 & -0.2 & 31.9 & 31.3 & -0.6 \\
ABD 1B & 85.8 & 85.6 & -0.2 & 85.8 & 85.6 & -0.2 \\
ABD 1C & 145.2 & 145.5 & 0.3 & 89.5 & 88.8 & -0.7 \\
ABD 1D & 157.6 & 156.9 & -0.7 & 87.7 & 87.9 & 0.2 \\
\hline
\end{tabular}

${ }^{a}$ All of the ranges and modulation widths were calculated in a water phantom of $\mathrm{D}_{90}$.

${ }^{b}$ The ABD $1 \mathrm{~A}$ is a tomotherapy beam field which is not included in this study.

(a)

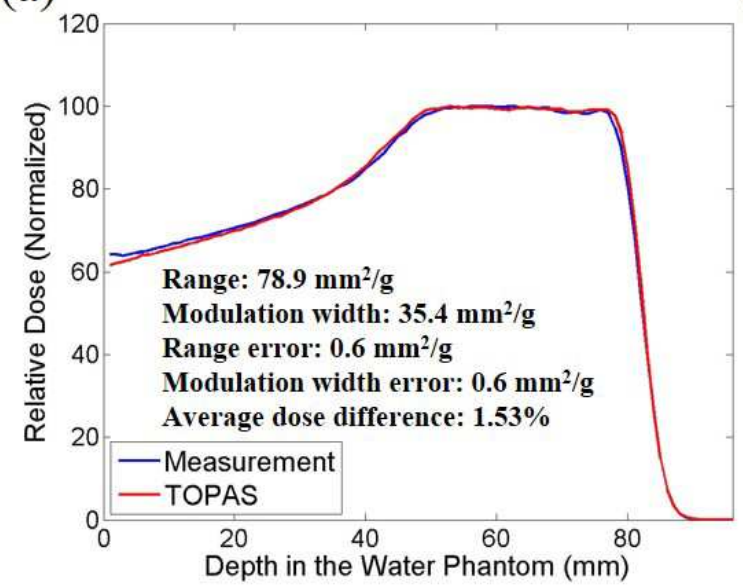

(b)

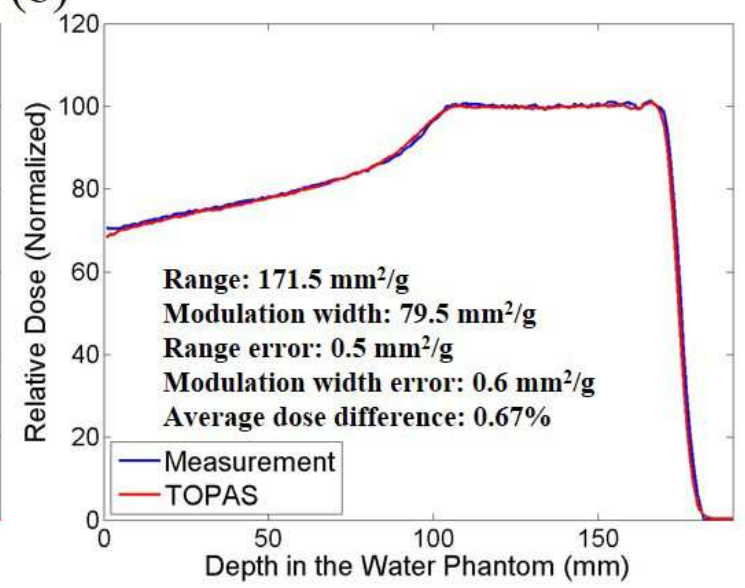

Figure 5. PDDs measured with a Markus ionization chamber (blue) and calculated with the TOPAS system (red), and the errors between them for the range and the modulation width of the B3 (a) and the B6 option (b).

a supercomputer called TACHYON, supported by the Korea Institute of Science and Technology Information (KISTI) (Ahn et al. 2014). The calculated dose distributions were compared with the RTP dose distributions in terms of dose difference (DD), 3D gamma index, and dose volume histograms (DVHs) using MATLAB and an in-house developed code. 
(a)

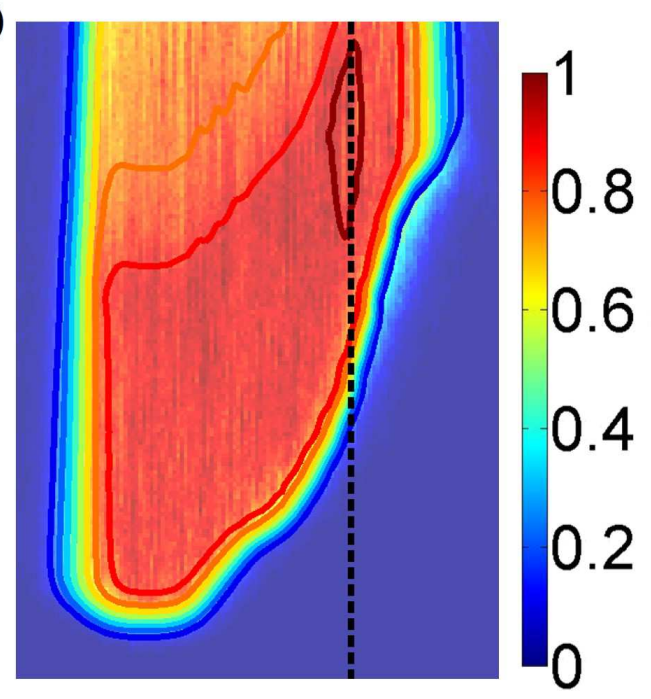

(b)

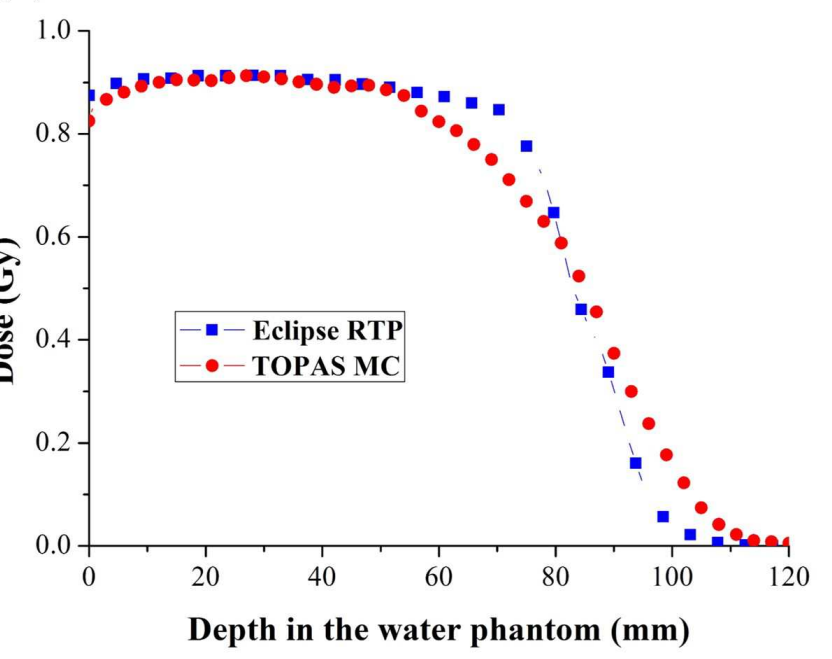

Figure 6. The RTP dose distribution (contour) and the MC dose distribution (color distribution) in the virtual water phantom with the compensator of ABD 1C (a), and the dose profile calculated with the RTP (blue squares) and MC (red circles) along the black dashed line in (a) representing the sharp slope of the compensator (b).

\section{Results and discussions}

\subsection{Validation of the proton beam and PSCs}

Figure 5 shows examples of PDDs calculated with the developed beam nozzle code in the virtual water phantom and PDDs empirically measured with the Markus ionization chamber using the eight beam options of the beam nozzle at the KNCC for beam commissioning (listed in Table 1). The primary protons $\left(10^{7}\right)$ were simulated and a calculation time of 10 CPU-hours was required for each beam option. The virtual water phantom was divided into $1-\mathrm{mm}$ bins along the longitudinal direction. With a spline interpolation, the difference between the MC-simulations and measurements was assessed to be smaller than $0.8 \mathrm{~mm}^{2} / \mathrm{g}$ for both the range and modulation width. Two cases of the results were shown in figure 6 with the B3 and B6 options, respectively. The average dose differences were assessed less than $2 \%$ in all depth bins. The empirically calibrated zero angles of the RMW were -5.6 to $5.6 \mathrm{deg}$, depending on the RMW track. The beam commissioning demonstrated good agreement within the tolerance of the QA measurement with an accuracy of $1 \mathrm{~mm}^{2} / \mathrm{g}$ for the range and modulation width, and the results reflected the accuracy of the beam modeling.

Figure 6(a) shows the dose distributions that were calculated with the MC method and the RTP in the virtual water phantom to validate the automatically modeled aperture and compensator. The dose matrix size of both the RTP and MC was 0.15625 $\times 0.15625 \times 0.25 \mathrm{~cm}^{3}$, and the number of voxels was $192 \times 192 \times 120$. The field size determined by the aperture coordinator based on the lateral penumbra region (D50) shows good agreement between the RTP and the MC calculations within a voxel bin 


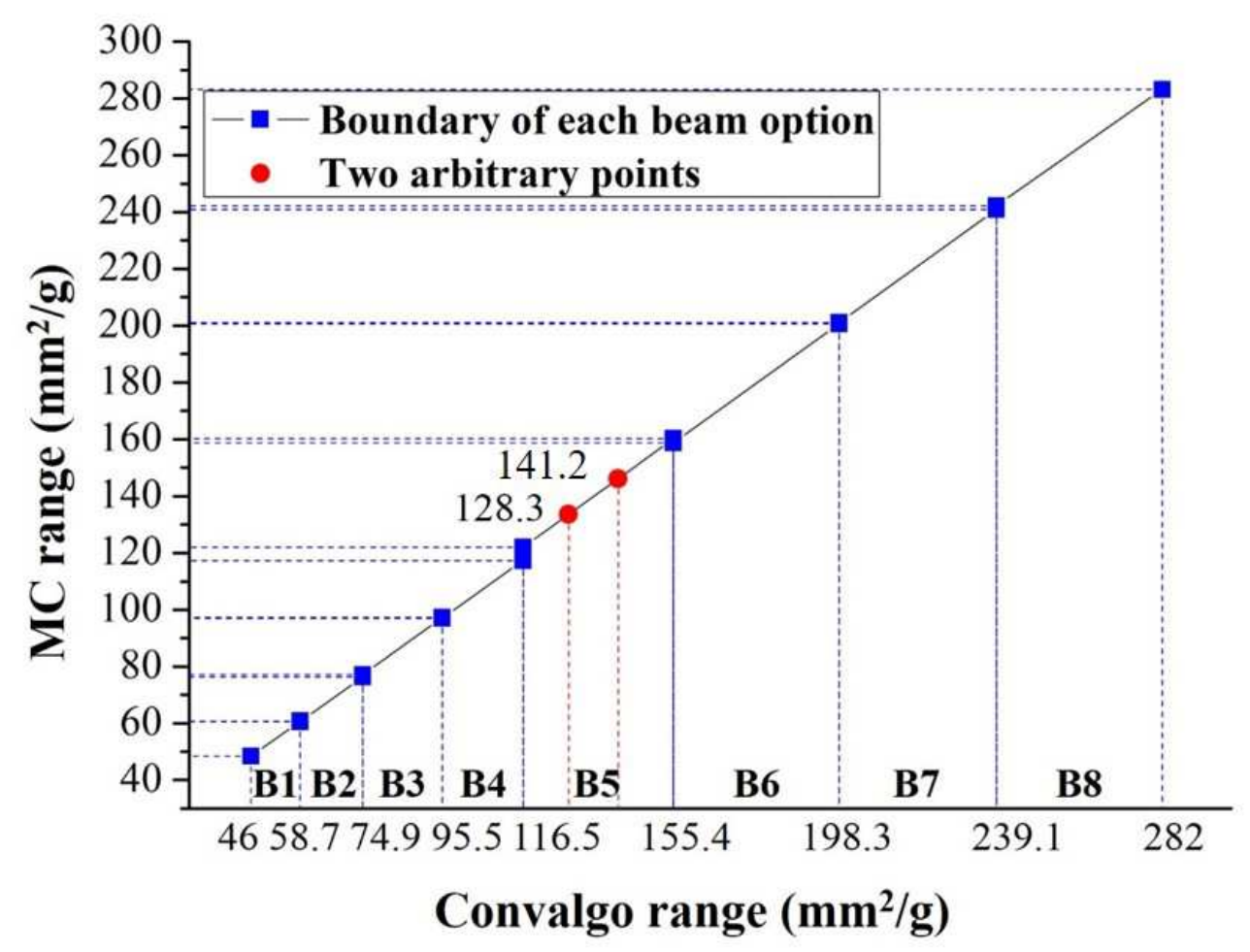

Figure 7. Calculated $\mathrm{MC}$ range at the maximum and the minimum of the eight beam options (blue squares), two arbitrary points for the B5 option (red circles), and the fitting curve of the relationship between the Convalgo range and the $\mathrm{MC}$ range, based on a linear interpolation (black line).

of $1.5625 \mathrm{~mm}$. However, a range degradation with a sharp slope of the compensator was observed after a depth of $6 \mathrm{~cm}$, as shown in figure 6(b). Despite the effect of the range degradation, the MC dose distribution was consistent with the RTP one, with a 3D gamma passing rate of $99.34 \%$, a distance to agreement (DTA) of $3 \mathrm{~mm}$, and a dose difference of $3 \%$. This validation ensured that the proton beam and PSCs were successfully modeled and the dose difference in the patient study might be caused by the well-known limitations of the PBA, such as the scatter disequilibrium in inhomogeneous regions, the range degradation in complex geometries, and the small field effect.

\subsection{Determination algorithm for beam set-up}

Figure 7 shows the range calibration, which was based on the correlation between the $\mathrm{MC}$ range and the Convalgo range. In the minimum and maximum ranges calculated with the TOPAS system, the difference between the range predicted by Convalgo and the $\mathrm{MC}$ range was observed to be up to $4 \mathrm{~mm}^{2} / \mathrm{g}$ in the $\mathrm{B} 5$ option owing to the simple range prediction of the Convalgo. However, the Convalgo range and the $\mathrm{MC}$ range showed a linear correlation within the boundary of each beam option; for example, two 
(a)

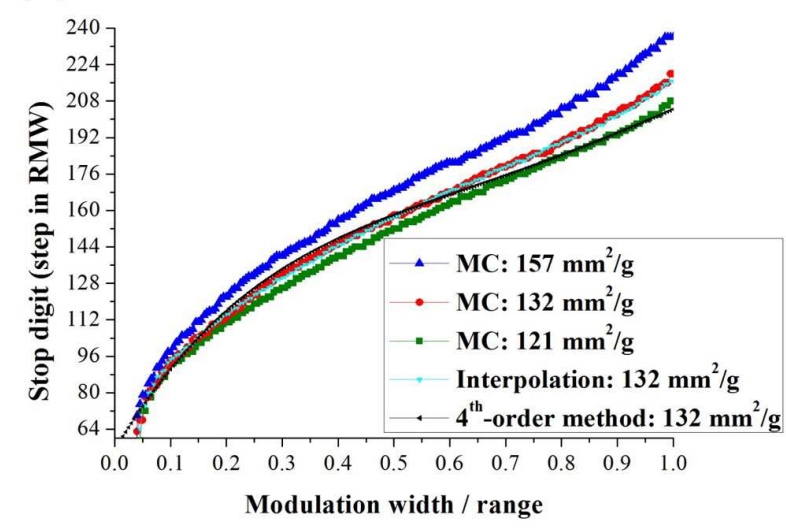

(b)

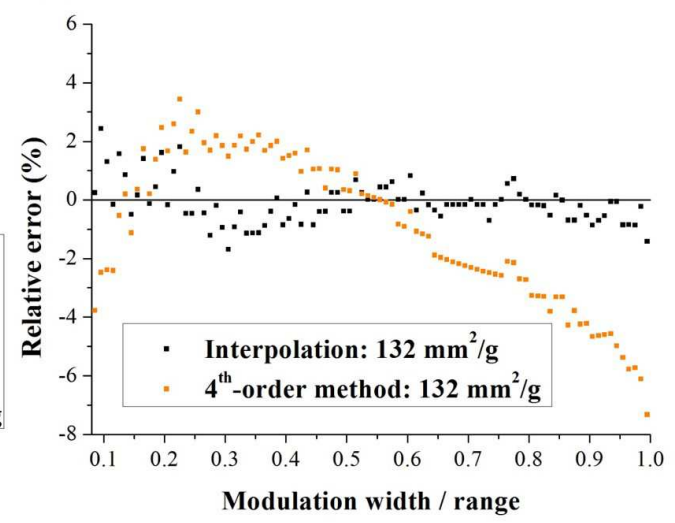

Figure 8. The stop-digit-to-modulation-width (SD-MW) curves for the B5 option in the proton range of 121 (green squares) and $157 \mathrm{~mm}^{2} / \mathrm{g}$ (blue triangles), and 132 $\mathrm{mm}^{2} / \mathrm{g}$ (red circles) calculated with the MC method, the interpolation method (cyan inverted triangles), and the $4^{\text {th }}$-order method (black left triangles) (a). Relative error of the interpolation method and the $4^{t h}$-order method provided by Convalgo with the reference $\mathrm{MC}$ results for the same proton range of $132 \mathrm{~mm}^{2} / \mathrm{g}$ (b).

arbitrary points of the 128.3 and $141.2 \mathrm{~mm}^{2} / \mathrm{g}$ ranges in the $\mathrm{B} 5$ option were calculated and the range difference was assessed to be up to $0.081 \mathrm{~mm}^{2} / \mathrm{g}$ for both ranges.

It must be noted that in the case of the modulation width, the stop digit determines the RMW step and the following modulation width. The single bin of the RMW angle was $1.40625 \mathrm{deg}$ for a discrete rotation of 256 steps for each beam option. Here, we suggest an interpolation method to accurately determine the stop digit, as explained in detail in the methods section. The stop-digit-to-modulation-width (SD-MW) curves normalized to each range should exhibit similar tendencies for the same beam option. Therefore, a specific stop digit could be assessed by interpolating the validated SDMW curves. In the current study, a database of SD-MW curves was constructed using the TOPAS system. The number of primary protons was $10^{9}$ and 540 CPU-hours were required to construct the modulation width database. For the validation of the interpolation method, the SD-MW curve of $132 \mathrm{~mm}^{2} / \mathrm{g}$ range was interpolated from the known reference SD-MW curves of 121 and $157 \mathrm{~mm}^{2} / \mathrm{g}$ range calculated with the $\mathrm{MC}$ method. The $4^{\text {th }}$-order function curve employed in Convalgo and the interpolation curve were compared with the SD-MW curve calculated with the MC method, which is considered as the reference, as shown in figure 8 (a). The SD-MW curve of the $4^{\text {th }}$-order function shows significant difference with the reference MC results with the relative errors up to $7.3 \%$, while the interpolation method shows the good relationship with the reference with average relative errors of $0.51 \%$.

\subsection{Case study}



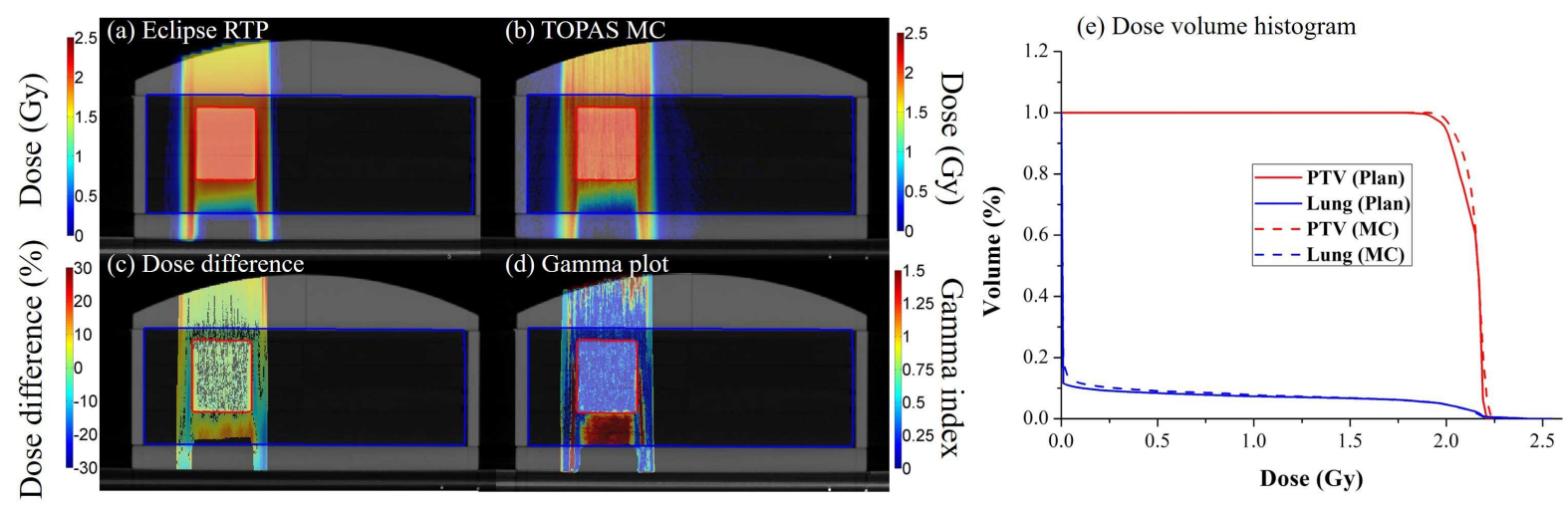

Figure 9. The results of the dose calculation in the lung phantom. The upper rows show the dose distribution calculated with Eclipse RTP (a) and TOPAS MC (b). The lower rows show the corresponding dose difference (c) and gamma plot (d). DVHs calculated using Eclipse RTP (solid line) and TOPAS MC (dashed line) (e). The red and blue contours and line indicate the acrylic PTV and the cork lung, respectively.

3.3.1. Lung phantom case The lung phantom was composed of acrylic plates, cork slabs, and pig bones representing the body, lung, and ribs, respectively. The planning target volume (PTV) was defined with the $3 \mathrm{~mm}$ margin from the target volume of three acrylic boxes of $5 \times 2 \times 5 \mathrm{~cm}^{3}$. The treatment plan was obtained by Eclipse RTP with the anterior-posterior (AP) beam, and the prescribed dose was 2 Gy to the PTV with $48.48 \mathrm{~mm}$ average aperture radius and $51.36 \mathrm{~mm}$ maximum compensator thickness. The MC dose calculation was performed using a computational phantom based on CT images of $512 \times 512 \times 90$ slices with voxel dimensions of $0.7227 \times 0.7227 \times 4 \mathrm{~mm}^{3}$. The acrylic body and target were converted to an arbitrary relative stopping power (RSP) of 1.08 and the cork lung was converted to a RSP of 0.22 . The dose distribution calculated with the MC method was compared with the planned dose, and the dose difference and $3 \mathrm{D}$ gamma index are shown in figure 9 . We evaluated a $3 \mathrm{D}$ gamma passing rate of 99.84\% in the PTV and 99.09\% in the lung. The distal range error in the water shows agreement within $0.5 \mathrm{~mm}^{2} / \mathrm{g}$; however, the physical range error was assessed as $2.4 \mathrm{~mm}$ in the cork lung of the low density. The range degradation in the dose fall-off region is observed, which appears like the well-known problem of the RTP caused by the sharp gradient of compensator (Paganetti 2011). Nevertheless, the DVHs in the PTV and the lung region show similar curves with the MC and the RTP which are implemented with the entirely different calculation process in figure $9(\mathrm{e})$.

\subsection{2. $A B D$ case A female patient was treated with tomotherapy for a squamous cell} carcinoma that occurred with the recurrence of cervical cancer and the tumor volume was boosted by proton therapy. The prescribed dose was 16.8 Gy to the PTV in seven fractions, and three beam fields of AP, right anterior oblique (RAO), and left anterior oblique (LAO) with the dose of 5.6 Gy were used. The average aperture radii were 44.49 $\mathrm{mm}, 49.84 \mathrm{~mm}$, and $47.04 \mathrm{~mm}$, and the maximum compensator thicknesses were 49.05 

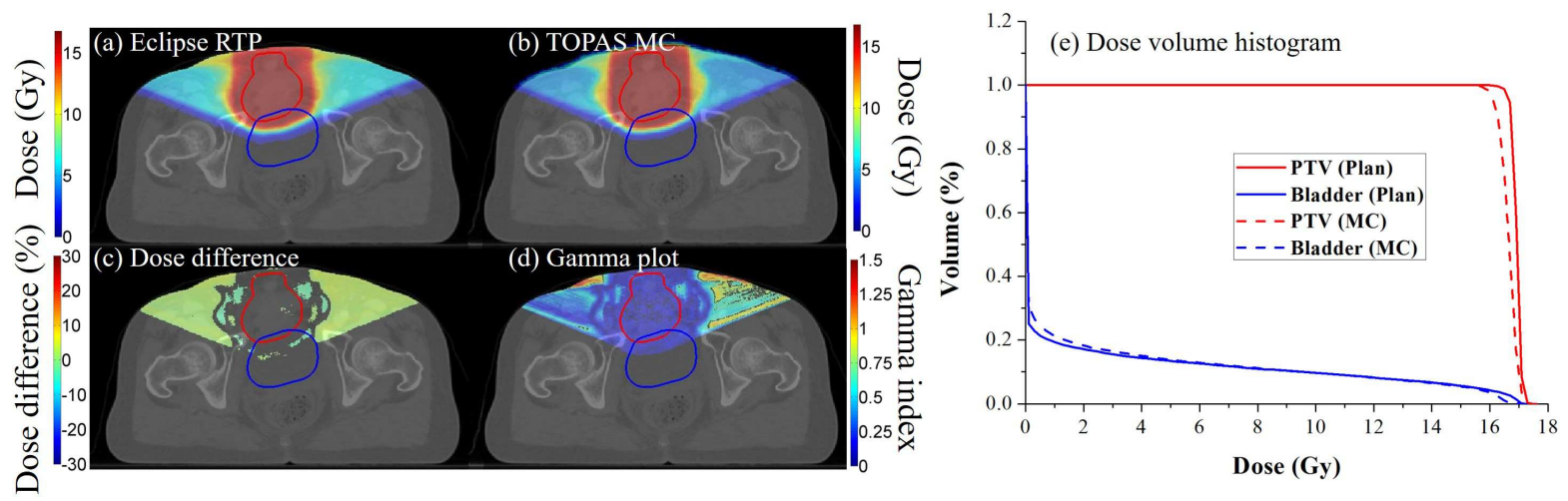

Figure 10. The results of the dose calculation in the ABD case. The upper rows show the dose distribution calculated with Eclipse RTP (a) and TOPAS MC (b). The lower rows show the corresponding dose difference (c) and gamma plot (d). DVHs calculated using Eclipse RTP (solid line) and TOPAS MC (dashed line) (e). The red and blue contours and line indicate the PTV and the bladder, respectively.

$\mathrm{mm}, 106.75 \mathrm{~mm}$, and $112.30 \mathrm{~mm}$, respectively, for each beam field. The number of CT voxels was $512 \times 512 \times 159$ and the voxel size was $0.8789 \times 0.8789 \times 3 \mathrm{~mm}^{3}$. The proton beam field contains the bladder, sigmoid colon, rectum, and urethra. The treatment plan needed to be verified especially for the colon because of its radio-sensitivity.

Figure 10 shows the dose distributions calculated with the MC method and the RTP. The dose agreement was evaluated using the dose difference and the 3D gamma index in the same conditions as those used in the lung phantom study. The dose distributions calculated with the MC using the complicated process shows almost the identical with that by RTP. Out of the target, the dose difference of less than $3 \%$ was observed with the RAO and LAO beams. At the fall-off region of each beam, several notable differences between MC and RTP dose were shown around the target in dose difference plot. These discrepancies are evaluated with the scattering effect in the compensator due to the sharp gradient of the compensators. However, the current results with the homogeneous target indicate good agreement between the MC and RTP dose calculations with a $99.97 \%$ 3D gamma passing rate for the PTV and $99.98 \%$ for the bladder. Figure 10(e) shows the DVHs for the PTV and bladder assessed with the MC method and the RTP. The PTV and bladder results showed close similarity in the DVH. The volume fraction of low-dose regions in the bladder calculated by the MC method were higher than those calculated with the RTP by up to $5 \%$. Moreover, the gradual curve was observed at the fall-off of the PTV (MC) due to the effect of the statistical errors (Keall et al. 2000). The results for the homogeneous case indicate that the dose calculated with the MC method is good enough for the independent validation against the RTP. It is potentially possible that their differences in the heterogeneous region and in the region of the different density could come from the limited dose calculation algorithm of the RTP. 

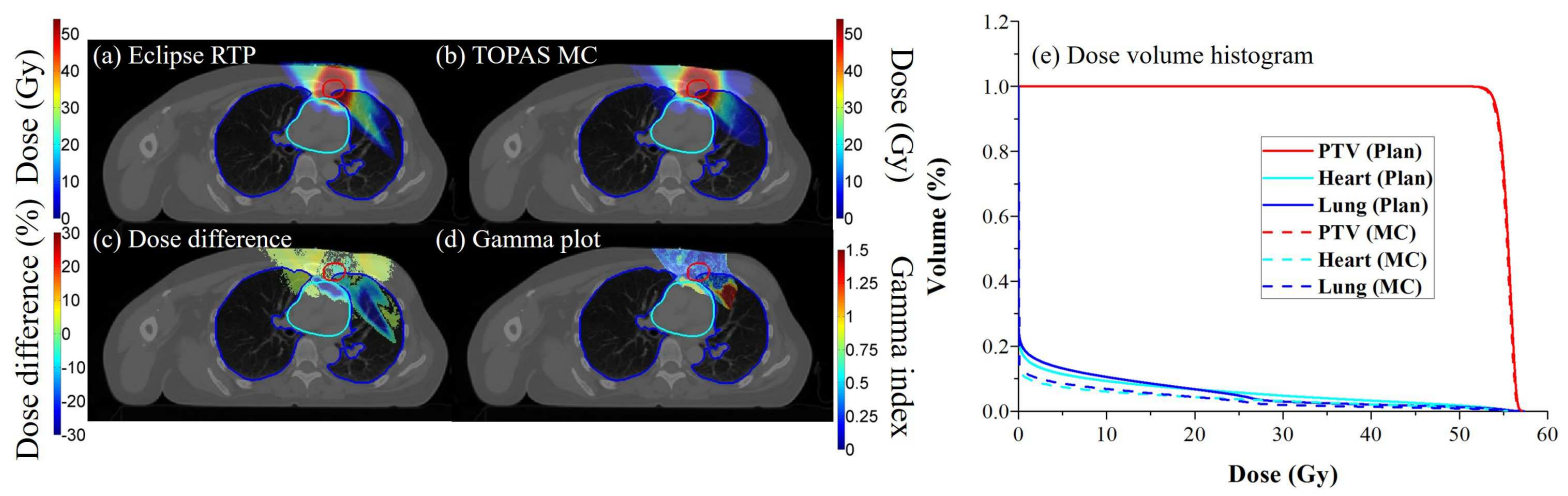

Figure 11. The results of the dose calculation in the IMN case. The upper rows show the dose distribution calculated with Eclipse RTP (a) and TOPAS MC (b). The lower rows show the corresponding dose difference (c) and gamma plot (d). DVHs calculated using Eclipse RTP (solid line) and TOPAS MC (dashed line) (e). The red, cyan, and blue contours and line indicate the PTV, heart, and lung, respectively.

3.3.3. IMN case A female patient was treated for a ductal carcinoma. The prescribed dose to the PTV was 54 Gy in 20 fractions with RAO and LAO beams. The RAO and LAO beams were planned with average aperture radii of 47.51 and $41.83 \mathrm{~mm}$ and maximum compensator thicknesses of 55.71 and $47.83 \mathrm{~mm}$, respectively. This case includes a fiducial marker positioned at the front of the sternum, and the HU values of the marker was assigned to the RSP of 0 because it would be removed from the patient during radiation delivery. The HU values of the heart was also assigned to a RSP of 1.05 with the consideration of the compensation for the vascular contrast media based on the protocol of the KNCC. The contrast media in the lung vessels also would be removed in the clinic, however, HU assignment was not applied because the vessels beyond the target dont contribute the target dose. The MC dose was calculated based on a CT phantom with $512 \times 512 \times 97$ slices with voxel dimensions of $0.9766 \times 0.9766 \times 3.75$ $\mathrm{mm}^{3}$. The accuracy of the dose calculation in the lung site was important because the dose difference between the RTP and MC calculations has been reported to be significant near the lung because of inhomogeneities.

The results of the IMN case showed a 3D gamma passing rate of $99.79 \%$ for the PTV, $98.36 \%$ for the heart, and $98.55 \%$ for the lung, as shown in figure 11 . Even if the target was clearly covered with the MC and RTP, the significant dose difference was observed in the lung region. Here, the beam range with the MC shows much less than that by RTP. Notice that the beam range of the MC and RTP is identical in water phantom. To quantitatively analyze the reason of difference, the dose profile in the isocenter was illustrated with the CT value in figure 12. Near the dose fall-off region, the $\mathrm{MC}$ dose decreased in lung vessels of the relatively high $\mathrm{HU}$ values in the lung region. The final ranges of both the MC and RTP were almost similar, while the MC dose distribution shows two times of the steep drop near the lung vessels. It is possible that the contrast agents enhanced the HU value of the lung vessels, while the RTP 


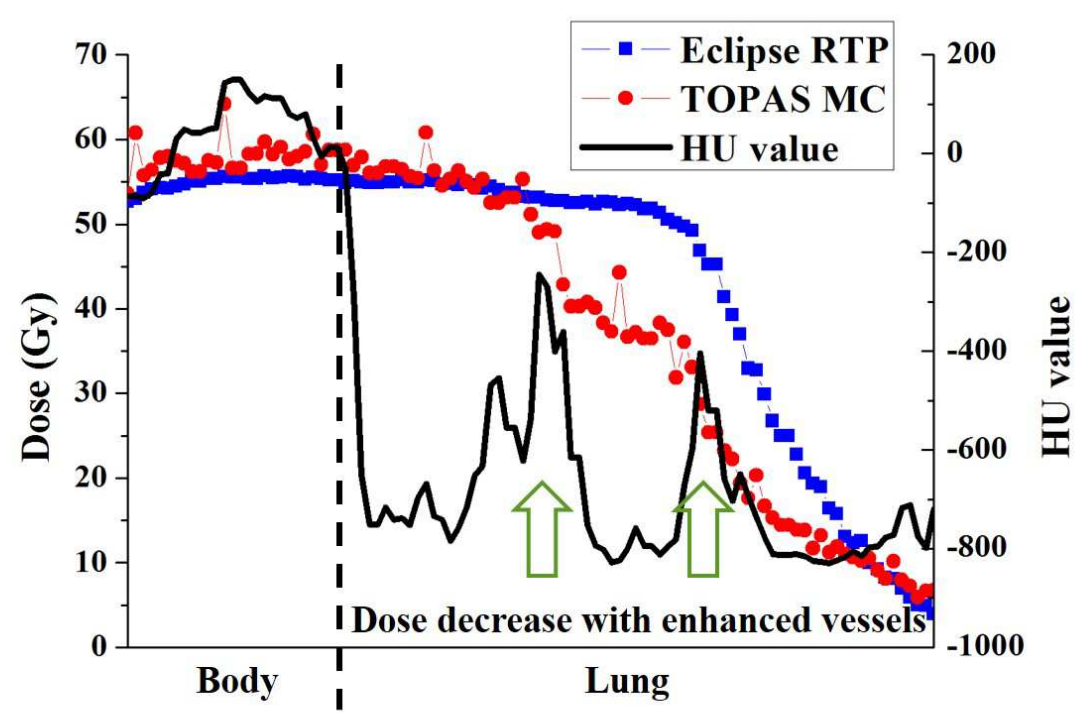

Figure 12. Dose profile in the isoplane for the IMN case. The discrepancies between the Eclipse RTP (blue squares) and TOPAS MC (red circles) are observed with the enhanced lung vessels (green arrows).

employing the convolution method was limited in the calculation of the scattering effect caused by small inhomogeneous materials, such as lung vessels (Ahnesjö et al. 1992). This overdose in treatment plan would represent the less danger than the opposite case delivering the actual high dose to the critical organs and causing the side effect, however the overdose in the lung region could impose the suboptimal treatment plan. Figure 11(e) shows the DVH for the PTV, lung, and heart in the IMN case. The MC method predicted an identical agreement for the DVH in the PTV. However, significant differences of up to $8 \%$ were observed in the case of the lung because of the emphasis of the scattering effect in the lung vessels. For all that, those DVH curves still maintained the similar tendency with the DVH curve for the RTP plan dose.

\section{Conclusion}

In this study, we demonstrate that the MC method could be practically used for the independent dose validation tool in proton therapy. The passive scattering mode of the IBA universal nozzle installed at the KNCC was modeled using the TOPAS MC toolkit, and a determination algorithm was developed for the beam set-up to accurately simulate the SOBP with the precise range, modulation width, and output factor based on the automatic import of patient DICOM files. The validation of the MC calculation system was implemented by comparing the MC results with the planned dose. The developed MC system is currently used for the recalculation of special cases that require high-accuracy dose prediction for inhomogeneous materials (lung or head and neck 
cases) at the KNCC. Despite the complex process of the MC dose calculation, the dose distribution of the MC method and RTP showed good agreement for the phantom case, ABD case, and IMN case. However, we observed a partial dose difference case of the inhomogeneous region, a steep gradient of the range compensator, and limitations of the convolution method in the scattering calculation. Our results emphasize on the importance of the independent dose validation system using the MC to avoid of the overdose in the critical organ and suboptimal treatment plan. Depending on the position of the dose discrepancy, the safety margin could be modified and a suboptimal treatment plan could be employed to avoid of the overdose in the critical organ. To reduce the computational burden employing the MC dose calculation system, a variance reduction technique based on particle splitting can be applied at the range compensator (Ramos-Méndez et al. 2013). In addition, a graphic user interface linked with the patient management system at the KNCC will be developed for the clinical use.

\section{Acknowledgments}

This research was supported in part by grants from the National Cancer Center, Korea (NCC-1610340-1) and the Basic Science Research Program through the National Research Foundation of Korea (NRF) funded by the Ministry of Science, ICT \& Future Planning (2014R1A1A1007789).

\section{References}

Agostinelli S, Allison J, Amako K a, Apostolakis J, Araujo H, Arce P, Asai M, Axen D, Banerjee S, Barrand G et al. 2003 Geant4a simulation toolkit Nucl. Instrum. Methods Phys. Res. A 506(3), 250-303.

Ahn B Y, Jang J H, Ahn S I, Kim M I, On N R, Hong J H \& Lee S 2014 Study of high performance computing activation strategy Int. J. Multimedia and Ubiquitous Eng. 9(6), 59-66.

Ahnesjö A 1989 Collapsed cone convolution of radiant energy for photon dose calculation in heterogeneous media Med. Phys. 16(4), 577-592.

Ahnesjö A, Saxner M \& Trepp A 1992 A pencil beam model for photon dose calculation Med. Phys. 19(2), 263-273.

Almond P R, Biggs P J, Coursey B M, Hanson W, Huq M S, Nath R \& Rogers D 1999 Aapm's tg-51 protocol for clinical reference dosimetry of high-energy photon and electron beams Med. Phys. 26(9), 1847-1870.

Beltran C, Jia Y, Slopsema R, Yeung D \& Li Z 2014 A simplified methodology to produce monte carlo dose distributions in proton therapy J. App. Clin. Med. Phys. 15(4).

Berger M 1999 Stopping-power and range tables for electrons, protons, and helium ions.

Cheng P 2008 Cirs multi-modality phantom-ct image.

Cheong K H, Suh T S \& Cho B C 2004 The effects of the statistical uncertainties in monte carlo photon dose calculation for the radiation therapy J. Radiat. Prot. Res. 29(2), 105-115.

Enger S A, Landry G, D'Amours M, Verhaegen F, Beaulieu L, Asai M \& Perl J 2012 Layered mass geometry: a novel technique to overlay seeds and applicators onto patient geometry in geant4 brachytherapy simulations Phys. Med. Biol. 57(19), 6269.

Jarlskog C Z \& Paganetti H 2008 Physics settings for using the geant4 toolkit in proton therapy IEEE Trans. Nucl. Sci. 55(3), 1018-1025. 
Jia X, Gu X, Sempau J, Choi D, Majumdar A \& Jiang S B 2010 Development of a gpu-based monte carlo dose calculation code for coupled electron-photon transport Phys. Med. Biol. 55(11), 3077.

Keall P, Siebers J, Jeraj R \& Mohan R 2000 The effect of dose calculation uncertainty on the evaluation of radiotherapy plans Med. Phys. 27(3), 478-484.

Kim D W, Lim Y K, Ahn S H, Shin J, Shin D, Yoon M, Lee S B, Kim D Y \& Park S Y 2011 Prediction of output factor, range, and spread-out bragg peak for proton therapy Med. Dosi. 36(2), 145-152.

Kooy H M, Rosenthal S J, Engelsman M, Mazal A, Slopsema R L, Paganetti H \& Flanz J B 2005 The prediction of output factors for spread-out proton bragg peak fields in clinical practice Phys. Med. Biol. 50(24), 5847.

Lee S K, Kim Y N \& Kim S K 2015 A feasibility study on using neural network for dose calculation in radiation treatment J. Radiat. Prot. Res. 40(1), 55-64.

Lin L, Shen J, Ainsley C G, Solberg T D \& McDonough J E 2014 Implementation of an improved doseper-mu model for double-scattered proton beams to address interbeamline modulation width variability J. Appl. Clinical Med. Phys. 15(3), 297-306.

Martin J 2016 'Particle therapy patient statistics (per end of 2015)'. URL: $\quad h t t p \quad: \quad / / w w w . p t c o g . c h / a r c h i v e /$ patient $_{\text {statistics/Patientstatistics - }}$ updateDec2015.pdf

MathWorks I 2005 MATLAB: the language of technical computing. Desktop tools and development environment, version 7 Vol. 9 MathWorks.

Méndez J R, Perl J, Schümann J, Shin J, Paganetti H \& Faddegon B 2015 Improved efficiency in monte carlo simulation for passive-scattering proton therapy Phys. Med. Biol. 60(13), 5019.

NEMA 2009 Digital imaging and communications in medicine (DICOM) NEMA.

Paganetti H 2009 Dose to water versus dose to medium in proton beam therapy Phys. Med. Biol. $\mathbf{5 4}(14), 4399$.

Paganetti H 2011 Proton therapy physics CRC Press.

Paganetti H 2012 Range uncertainties in proton therapy and the role of monte carlo simulations Phys. Med. Biol. 57, R99-117.

Paganetti H, Jiang H, Parodi K, Slopsema R \& Engelsman M 2008 Clinical implementation of full monte carlo dose calculation in proton beam therapy Phys. Med. Biol. 53(17), 4825.

Perl J, Shin J, Schümann J, Faddegon B \& Paganetti H 2012 Topas: an innovative proton monte carlo platform for research and clinical applications Med. Phys. 39(11), 6818-6837.

Rah J E, Shin D, Oh D H, Kim T H \& Kim G Y 2014 Feasibility study of using statistical process control to customized quality assurance in proton therapy Med. Phys. 41(9).

Ramos-Méndez J, Perl J, Faddegon B, Schümann J \& Paganetti H 2013 Geometrical splitting technique to improve the computational efficiency in monte carlo calculations for proton therapy Med. Phys. 40(4).

Schneider U, Pedroni E \& Lomax A 1996 The calibration of ct hounsfield units for radiotherapy treatment planning Phys. Med. Biol. 41(1), 111.

Schneider W, Bortfeld T \& Schlegel W 2000 Correlation between ct numbers and tissue parameters needed for monte carlo simulations of clinical dose distributions Phys. Med. Biol. 45(2), 459.

Shin J, Kim D, Lim Y K, Ahn S, Shin D, Yoon M G, Park S Y, Lee S B, Kwak J \& Son D 2010 Monte carlo modeling and simulation of a passive treatment proton beam delivery system using a modulation wheel J. Korean Phys. Soc. 56(1), 153-163.

Shin W G, Min C H, Shin J I, Jeong J H \& Lee S B 2015 A monte carlo study of the relationship between the time structures of prompt gammas and the in-vivo radiation dose in proton therapy J. Korean Phys. Soc. 67(1), 248-253.

Testa M, Schümann J, Lu H M, Shin J, Faddegon B, Perl J \& Paganetti H 2013 Experimental validation of the topas monte carlo system for passive scattering proton therapy Med. Phys. 40(12).

Van Esch A, Tillikainen L, Pyykkonen J, Tenhunen M, Helminen H, Siljamäki S, Alakuijala J, Paiusco M, Iori M \& Huyskens D P 2006 Testing of the analytical anisotropic algorithm for photon dose calculation Med. Phys. 33(11), 4130-4148. 
Independent dose verification system with Monte Carlo simulations using TOPAS 21

Verburg J M, Grassberger C, Dowdell S, Schuemann J, Seco J \& Paganetti H 2016 Automated monte carlo simulation of proton therapy treatment plans Technol. Cancer Res. Treat. 15(6), NP35NP46.

White D, Woodard H \& Hammond S 1987 Average soft-tissue and bone models for use in radiation dosimetry British J. Rad. 60(717), 907-913. 